\title{
Theoretical shaping of femtosecond laser pulses for ultrafast molecular photo-dissociation with control techniques based on time-dependent density-functional theory
}

\author{
Alberto Castro *††
}

\section{Abstract}

The combination of time-dependent density-functional theory and the quantum optimal control formalism is used to optimize the shape of ultra-short laser pulses in order to achieve the photo-dissociation of the Hydrogen molecule. The very short duration of the pulses used in this work (a few femtoseconds) does not allow for significant nuclear movement during its action, and therefore the dissociation mechanism is sequential: during the pulse irradiation, a large sudden momentum is communicated - which can be understood in terms of population of excited, bound or not, dissociative electronic states. The target is defined in terms of the average opposing force during the action of the pulse, or equivalently in terms of the final dissociative velocity.

\section{Introduction}

Recently, the use of time-dependent density functional theory (TDDFT) [1, 2] was proposed to perform optimizations in electronic systems in combination with the quantum optimal control theory (QOCT)[3] methodology[4]. This scheme permits to theoretically study, from first principles, the optimization possibilities in the time-dependent many-electron manifold. Performing quantum dynamics for many-electron systems is a notoriously difficult computational task, and the use of TDDFT allows to reduce this difficulty by working with a simpler noninteracting system of electrons, whose time-dependent density is nevertheless

\footnotetext{
*ARAID Foundation - Institute for Biocomputation and Physics of Complex Systems (BIFI), University of Zaragoza, Mariano Esquillor s/n, Edificio I+D, 50018 Zaragoza (Spain)

${ }^{\dagger}$ Zaragoza Scientific Center for Advanced Modeling (ZCAM), Mariano Esquillor s/n, Edificio I+D, 50018 Zaragoza, Spain

${ }^{\ddagger}$ Unidad Asociada IQFR-BIFI, University of Zaragoza, Mariano Esquillor s/n, Edificio $\mathrm{I}+\mathrm{D}, 50018$ Zaragoza, Spain
} 
identical to the real one. In Ref. [4] the theoretical basis of the TDDFT+QOCT combination was established, and exemplified with a relatively simple example: the charge transfer in a model double quantum dot formed in a two-dimensional homogeneous electron gas. This work presents the first application of the scheme to the problem of photo-dissociation with realistic three-dimensional models of molecules.

Most of the previous applications of QOCT have targeted the nuclear dynamics - and in a sense, this work, that attempts to optimize photo-dissociation events, is also about nuclear dynamics. However, there is a fundamental change in the approach: in most previous applications the optimization objects are nuclear wave-packets, moving in one or various adiabatic potential energy surfaces. In this manner, the electrons are previously integrated out; in contrast, the TDDFT+QOCT formalism attempts to directly address and optimize the electronic motion.

This shift of focus from the nuclear to the electronic degrees of freedom is motivated by the growth of attosecond physics[5,6]. The possibility of creating laser pulses shorter than one femtosecond $[7,8]$ has given birth to a whole new area of experimental physics in which the electronic motion can be directly followed - and controlled - in its natural time-scale. Novel experimental techniques such as attosecond transient absorption spectroscopy, or attosecond time-resolved photo-electron spectroscopy allow for this possibility, and TDDFT should play a fundamental role in the interpretation of these processes [9].

The frontier between femtosecond and attosecond physics is of course blurred, but some distinctive features are often present. For example, in femto-chemistry, the nuclear degrees of freedom are usually the main objective, and therefore the key frequencies are typically in the infrared region. In the attosecond realm, the frequencies should be increased to the XUV region in order to address the electronic motion, that is much faster. The capability of controlling a quantum process with an external field is related to the capability of shaping this field - the more freedom we have to tailor the laser pulse at will, the more control we will attain on the system. Extremely versatile laser shapers have been developed over the last decades [10], and great liberty has been achieved regarding durations, frequencies, or intensities. Outside this experimental attainable range, theoretical models may substitute experiments and help us understand how much the quantum systems can be controlled.

In this work, I take a first step in the exploration of the control possibilities of the electronic systems in atoms and molecules with the TDDFT+QOCT formalism developed in Ref. [4]. For a first step, the Hydrogen molecule is an appropriate example choice due to its simplicity. The control methodology addresses the electronic system only, and therefore the optimizations must be performed with clamped nuclei. This can only model interactions with very short pulses, so that the heavier nuclei do not have time to move significantly. Nevertheless, the target functional is chosen to achieve the molecular photodissociation. This apparent contradiction is not such since the dissociation is assumed to happen through a sequential mechanism: during the pulse irradiation, a large sudden momentum is communicated to the nuclei from the electrons 
excited by the laser pulse. Assuming a classical approximation for the nuclei, the target may then be defined in terms of the forces that act on them during the action of the pulse - which are density functionals, suitable for a TDDFT description. If the time-average of these forces are opposite for the two nuclei, then they will communicate a large opposing momentum that will afterwards lead to photo-dissociation.

The pulses must afterwards be tested with moving nuclei, in order to check wether they produce dissociation or not. This can be done by performing simulations with the excited-states non-adiabatic molecular dynamics formalism based on TDDFT and the Ehrenfest equations [11, 12, 13, 14, 15, 16].

The optimal control scheme has been implemented in the octopus package[17, 18]. Previous applications include the control of currents in quantum rings [19], the manipulation of charge in quantum dots [20], or the enhancement of molecular ionization [21]. Furthermore, in a previous work[22] we have already employed TDDFT in combination with optimization schemes to study optimal molecular photo-dissociation. However, in that case the optimizations on systems with interacting electrons described with TDDFT were not performed with the TDDFT+QOCT formalism, but rather by making use of a gradientless optimization algorithm, i.e. one scheme that only necessitates the repeated computation of the value of the function to be optimized with different control parameters, which can be obtained by successive forward propagations. The use of the full power of the optimal control formalism, as it is done in this work, significantly improves the optimization efficiency thanks to the extra information provided by the function gradient.

\section{Methodology}

The mathematical framework that results of the combination of TDDFT and QOCT was explained in Refs. [4] and [23]. Here I will display the main equations, and develop the general framework for the particular targets that have been used.

In analogy to the "traditional" ground state density-functional theory (DFT) [24], TDDFT establishes a one-to-one correspondence between densities and external potentials - only in this case they are both time-dependent objects. This implies that any system property is, in principle, a functional of the density. Likewise, TDDFT usually also employs a Kohn-Sham (KS) scheme[25], in which one utilizes a fictitious system of non-interacting electrons that has the same density of the real system. This strongly reduces the computational complexity, since non-interacting fermions can be described with a single Slater determinant. This problem substitution cannot however be performed exactly because the external potential acting on the non-interacting electrons must be approximated.

If we consider a spin-compensated system of $N$ electrons, we may construct the KS Slater determinant with $N / 2$ spatial orbitals $\varphi_{i}(i=1, \ldots, N / 2)$. Let $v(\vec{r}, t)$ be the external potential responsible for the irradiation of the real system, driving it from its initial ground state during an interval of time $[0, T]$. The 
"time-dependent Kohn-Sham" (TDKS) equations that characterize the evolution of the fictitious system are (atomic units will be used hereafter):

$$
\begin{aligned}
\mathrm{i} \frac{\partial}{\partial t} \varphi_{i}(\vec{r}, t) & =-\frac{1}{2} \nabla^{2} \varphi_{i}(\vec{r}, t)+v_{\mathrm{KS}}[n](\vec{r}, t) \varphi_{i}(\vec{r}, t), \\
\varphi_{i}(\vec{r}, 0) & =\varphi_{i}^{\mathrm{gs}}(\vec{r}), \\
n(\vec{r}, t) & =2 \sum_{i=1}^{N / 2}\left|\varphi_{i}(\vec{r}, t)\right|^{2},
\end{aligned}
$$

where $\left\{\varphi_{i}^{\mathrm{gs}}\right\}_{i=1}^{N / 2}$ are the ground state Kohn-Sham orbitals. Eq. (3) provides the time-dependent density $n(\vec{r}, t)$ in terms of the KS orbitals. The KS potential $v_{\mathrm{KS}}$ is a functional of this density, defined as:

$$
v_{\mathrm{KS}}[n](\vec{r}, t)=v_{0}(\vec{r} ; \underline{R})+v(\vec{r}, t)+v_{\mathrm{H}}[n](\vec{r}, t)+v_{\mathrm{xc}}[n](\vec{r}, t),
$$

where the Hartree potential $v_{\mathrm{H}}$ is given by:

$$
v_{\mathrm{H}}[n](\vec{r}, t)=\int \mathrm{d}^{3} r^{\prime} \frac{n\left(\vec{r}^{\prime}, t\right)}{\left|\vec{r}^{\prime}-\vec{r}\right|},
$$

and $v_{0}(\vec{r} ; \underline{R})$ is the static external potential that characterizes the system in its ground state; it is determined by the positions of a set of nuclei $\underline{R}=\vec{R}_{1}, \ldots, \vec{R}_{K}$. Finally, the "exchange and correlation" potential $v_{\mathrm{xc}}[n]$ is also a functional of the density. This object is in practice unknown and must be approximated[26]. In this work I will use the local density approximation (LDA) as parameterized in Ref. [27], by considering its adiabatic extension for time-dependent problems, which consists of using the same ground state functional for the time-propagated density at each moment. Unfortunately, this choice has well-known deficiencies, in particular for the dissociating molecular Hydrogen problem[28], which is in fact the example shown below. However, during the optimization process itself the nuclei are kept at their equilibrium positions, and therefore the error that should appear at long internuclear distances is not present. The dynamics that follows will however not be very accurate; in future works the use of better exchange and correlation choices needs to be explored.

The external potential $v(\vec{r}, t)$, in this case, is the electric field representing a laser pulse in the dipole approximation and in the length gauge:

$$
v(\vec{r}, t)=\varepsilon(t) \vec{r} \cdot \vec{p},
$$

where the unitary vector $\vec{p}$ determines the light polarization. The variation of its temporal dependence $\varepsilon(t)$ is our handle on the system, and constitutes the control mechanism. In general, we may consider its temporal shape to be determined by $M$ parameters $u \in \mathbb{R}^{M}$, and therefore both $\varepsilon$ and $v$ are in fact functions of $u$ : $\varepsilon(t)=\varepsilon[u](t), v(\vec{r}, t)=v[u](\vec{r}, t)$.

This is the external potential that drives the real system of interacting electrons, determining its evolution and therefore the time-dependent density, which becomes in this form a function of the control $u$ :

$$
u \longrightarrow n[u](\vec{r}, t) \text {. }
$$


Our goal is to find a set of parameters $u$ that drives the system in a certain "optimal" manner. This optimal behavior is mathematically defined as the maximization of a function in the form:

$$
G[u]=\tilde{F}[n[u]],
$$

where $\tilde{F}$ is a functional of the system evolution (in terms of its density). The precise form of the functional dependence of $\tilde{F}$ on the system evolution depends on our goal; below we will use one form designed to encourage bond breaking. Sometimes, the target functional also has an explicit dependence on $u$, that is used to constrain its shape according to physical considerations - e.g. impose a penalty on too high intensities or frequencies. However, in this work we will use a parameterization $\varepsilon=\varepsilon[u]$ that by definition constrains the pulse to have an admissible shape.

In principle, one can use a functional of the full system wave function, not only of its density. However, this definition permits to use TDDFT without further approximations: since the density of the real and of the KS system are identical, we may work with the latter. The theory is in this case developed in terms of a functional $F$ of the KS orbitals, which can be defined by simply substituting the density by its expansion in terms of the orbitals:

$$
G[u]=F[\underline{\varphi}[u]]=\tilde{F}[n[u]],
$$

where $\varphi[u]$ represents all the $N / 2$ Kohn-Sham orbitals, and its dependence on $u$ stresses the fact that they are also determined by the choice of the control parameters.

The gradient of $G$ can then be computed as[4]:

$$
\nabla G[u]=2 \sum_{j=1}^{N / 2} \int_{0}^{T} \mathrm{~d} t \nabla \varepsilon[u](t) \operatorname{Im}\left\langle\chi_{j}[u](t)|\hat{\vec{r}} \cdot \hat{p}| \varphi_{j}[u](t)\right\rangle .
$$

This expression uses an auxiliary set of orbitals $\left\{\chi_{j}[u]\right\}_{j=1}^{N / 2}$ defined by the following equations of motion:

$$
\begin{aligned}
\mathrm{i} \frac{\partial}{\partial t} \chi_{i}[u](\vec{r}, t)= & -\frac{1}{2} \nabla^{2} \chi_{i}[u](\vec{r}, t)+v_{\mathrm{KS}}^{*}[n[u]](\vec{r}, t) \chi_{i}[u](\vec{r}, t) \\
& +\sum_{j=1}^{N / 2} \hat{K}_{i j}[\underline{\varphi}[u](t)] \chi_{j}[u](\vec{r}, t) \\
& -i \frac{\delta F[\underline{\varphi}[u]]}{\delta \varphi_{i}^{*}[u](\vec{r}, t)} \\
\chi_{j}[u](\vec{r}, T)= & 0 .
\end{aligned}
$$

These equations are similar to the TDKS equations verified by the $\underline{\varphi}$ orbitals, except for the following: 
1. There is a new set of operators, $\hat{K}_{i j}[\underline{\varphi}[u][t]]$, which are given by:

$$
\begin{array}{r}
\hat{K}_{i j}[\underline{\varphi}[u](t)] \chi_{i}[u](\vec{r}, t)= \\
-4 \mathrm{i} \varphi_{i}[u](\vec{r}, t) \operatorname{Im} \int \mathrm{d}^{3} r^{\prime} \chi_{i}^{*}[u]\left(\vec{r}^{\prime}, t\right) f_{\mathrm{Hxc}}[n[u](t)]\left(\vec{r}, \vec{r}^{\prime}\right) \varphi_{j}[u]\left(\vec{r}^{\prime}, t\right),
\end{array}
$$

where $f_{\mathrm{Hxc}}$ is the so-called kernel of the Kohn-Sham Hamiltonian:

$$
f_{\mathrm{Hxc}}[n]\left(\vec{r}, \vec{r}^{\prime}\right)=\frac{1}{\left|\vec{r}-\vec{r}^{\prime}\right|}+\frac{\delta v_{\mathrm{xc}}[n](\vec{r})}{\delta n\left(\vec{r}^{\prime}\right)} .
$$

2. These propagating equations for $\chi[u]$ are inhomogeneous due to the presence of the last term in the right hand side of Eq. (11), which is the functional derivative of the target functional with respect to each of the orbitals.

3. The KS potential $v_{\mathrm{KS}}$ appears conjugated; usually this is irrelevant since one assumes it to be Hermitean. However, in order to deal, albeit approximately, with the possibility of ionization, one can use an imaginary potential at the borders of the simulation box, which will absorb the electronic density reaching those borders. This is used in the examples shown below.

4. The boundary condition for the differential equations are specified at the final propagation time (Eq. 12), instead of at the initial time. Note that this boundary condition is null; the reason for this is that the target functional that we will use in this work depends on the full evolution of the system, and has only an infinitesimal contribution from the final system state.

It remains to specify the form of the target functional. A discussion about the design of targets for the purpose of photo-dissociation was already given in Ref. [22]. Here I will use a definition in terms of the time-average of the forces $\vec{F}_{\alpha}(t)$,

$$
\vec{P}_{\alpha}=\int_{0}^{T} \mathrm{~d} t \vec{F}_{\alpha}(t),
$$

acting on the nuclei during the action of the pulse. This is convenient from a TDDFT perspective, since the forces are explicit functionals of the density:

$$
\vec{F}_{\alpha}(t)=\vec{F}_{\alpha}[n(t)]=-\int \mathrm{d}^{3} r n(\vec{r}, t) \vec{\nabla}_{\alpha} v_{0}(\vec{r} ; \underline{R}) .
$$

and therefore the averaged forces are likewise density functionals:

$$
\vec{P}_{\alpha}[n]=-\int_{0}^{T} \mathrm{~d} t \int \mathrm{d}^{3} r n(\vec{r}, t) \vec{\nabla}_{\alpha} v_{0}(\vec{r} ; \underline{R}) .
$$

The target is then defined in terms of a function of these averaged forces, $T=T\left(\vec{P}_{1}[n], \ldots, \vec{P}_{K}[n]\right)=T(\underline{P}[n])$. This function must be designed with 
the physical outcome that needs to be optimized in mind. For example, if the goal is to photo-dissociate a dimer, the definition may simply be:

$$
T(\underline{p}[n])=P_{1 x}[n]-P_{2 x}[n],
$$

where we assume that the two nuclei are originally positioned in the $x$ axis, and the laser polarization is likewise in that direction. This is the choice made for the example shown below, based on a simple intuition: if the nuclei are to dissociate, the forces exerted on them during a certain period of time must be opposite to each other.

In general,

$$
\tilde{F}[n]=T(\underline{P}[n]),
$$

and the necessary functional derivatives to be used in the equations of motion for $\underline{\chi}$ are:

$$
\frac{\delta F[\underline{\varphi}]}{\delta \varphi_{i}^{*}(\vec{r}, t)}=\sum_{\alpha=1}^{K} \sum_{\mu=x, y, z} 2 \frac{\partial T}{\partial P_{\alpha \mu}}(\underline{p}[n]) \frac{\partial v_{0}(\vec{r} ; \vec{R})}{\partial R_{\alpha \mu}} \varphi_{i}(\vec{r}, t) .
$$

Given the definition in Eq. (15) for $\vec{P}_{\alpha}$, and considering classical equations of motion for the nuclei, it seems natural to consider $\vec{P}_{\alpha}$ to be the variation of momentum of nucleus $\alpha$ (or, simply, the momemtum at time $T$ if initially it is at rest). However, let us recall that in the formalism described until now, the position of the nuclei are constrained to be static, and therefore this identification would only be an approximation.

This definition of the target functional is entirely based on the nuclear degrees of freedom: it does not, for example, prevent or encourage ionization of the system. In consequence, the dissociation of the system may be accompanied by a certain degree of electron loss. Note, however, that within this same scheme the ionization can also be controlled, either to enhance it [21] or to suppress it [29]. This could be done in this case by adding to the definition of the target, Eq. 19, a term dependent on the total density charge enclosed in a volume $V$ defined in the vicinity of the ions, multiplied by a weight whose sign would determine whether the ionization is desired or avoided, i.e.:

$$
\tilde{F}[n]=T(\underline{P}[n])+w \int_{V} \mathrm{~d}^{3} r n(\vec{r}, T) .
$$

In a more ambitious scheme, the final charge state of the fragments could also be controlled by making this term dependent separately on the charge surrounding each of these fragments.

In order to perform the optimization, I have used here the standard BroydenFletcher-Goldfarb-Shanno conjugate-gradients algorithm [30], as implemented in the GSL mathematical library [31]. Once the optimization is performed, it remains, however, to test whether or not the optimized pulse does actuaclly lead to photo-dissociation. In order to do that test, the clamped nuclei approximation needs to be lifted, and the $\vec{P}_{\alpha}$ vectors acquire their true meaning as nuclear 
momenta. The simulation is the performed with the Ehrenfest-TDDFT formalism, that consists of coupling, to the TDKS equations, the classical Hamiltonian equations of motion for the nuclear degrees of freedom:

$$
\begin{aligned}
\frac{\mathrm{d}}{\mathrm{d} t} \vec{R}_{\alpha}(t) & =\frac{\vec{P}_{\alpha}(t)}{M_{\alpha}}, \\
\frac{\mathrm{d}}{\mathrm{d} t} \vec{P}_{\alpha}(t) & =-\int \mathrm{d}^{3} r n(\vec{r}, t) \vec{\nabla}_{\alpha} v_{0}(\vec{r} ; \underline{R}(t)) .
\end{aligned}
$$

\section{Results}

The former methodology is here applied to the problem of finding the optimal pulse for the photo-dissociation of the Hydrogen molecule. The scheme has been implemented in the octopus code $[17,18,32]$. Here I will briefly describe the numerical parameters chosen for the simulations described below - the reader may find a detailed description on the numerical scheme in the above referenced articles.

The Hydrogen molecule initial bond-length is set to 1.44 a.u. The nucleuselectron interaction is not purely Coulombic, but rather it is softened through the pseudo-potential method [33]. This is necessary since wave functions, densities, potentials, etc., are represented on a real-space regular cubic mesh. The grid spacing is chosen to be 0.4 a.u., whereas the shape of the simulation box is a sphere of radius $13 \mathrm{a.u}$. This radius is doubled for the final Ehrenfest-TDDFT runs that test the performance of the optimized pulses in their bond-breaking task.

The duration of the laser pulses is fixed at $\approx 157$.a.u. $\approx 3.8 \mathrm{fs}$, which would correspond, e.g., to $25 \omega$ cycles of $\omega$ frequency (in atomic units). The fluence, or integrated intensity over the duration of the pulse, is defined as:

$$
F_{0}=\int_{0}^{T} \mathrm{~d} t \varepsilon^{2}(t)
$$

The choice of the search space is determined by this fluence: the search is performed only in the set of pulses whose fluence equals $F_{0}$ (a fully unconstrained search would in this case lead to a problem without an optimum, since it would allow pulses with unlimited intensities).

This is not, however, the only constraint: the pulse space is limited in frequencies. In order to simultaneously enforce these two constraints, I use here the procedure already described in Ref. [22]: First, the pulse temporal shape is expanded in a Fourier series:

$$
\varepsilon(t)=\sum_{i=1}^{2 L} \varepsilon_{i} g_{i}(t)
$$

where

$$
g_{i}(t)= \begin{cases}\sqrt{\frac{2}{T}} \cos \left(\frac{2 \pi}{T} i t\right) & (i=1, \ldots, L) \\ \sqrt{\frac{2}{T}} \sin \left(\frac{2 \pi}{T}(i-L) t\right) & (i=L+1, \ldots, 2 L)\end{cases}
$$


This fixes the maximum possible (cut-off), frequency to $\frac{2 \pi}{T} L$. Note that this explicitly omits the zero-frequency term, which is a desired restriction, in order to fulfill the physical condition

$$
\int_{0}^{T} \mathrm{~d} t \varepsilon(t)=0
$$

Another obvious condition that must be fulfilled is $\varepsilon(0)=\varepsilon(T)=0$, which implies:

$$
\sum_{i=1}^{L} \varepsilon_{i}=0 .
$$

In order to ensure this, we may work with a set of parameters $\left\{\alpha_{j}\right\}_{j=1}^{2 L-1}$, in terms of which the Fourier coefficients are given by:

$$
\begin{aligned}
\varepsilon_{1} & =-\sum_{j=1}^{L-1} \alpha_{j}, \\
\varepsilon_{i} & =\alpha_{j-1}(i=2, \ldots, 2 L)
\end{aligned}
$$

It is straightforward to prove that the fluence may then be written as:

$$
F_{0}=\alpha^{T} S \alpha,
$$

where $\alpha$ is the vector of $\alpha_{j}$ components, and $S$ is a $(2 L-1) \times(2 L-1)$ symmetric matrix. It may be diagonalized with the help of an orthonormal matrix $U$ :

$$
U^{T} S U=\operatorname{diag}\left(s_{1}, \ldots, s_{2 L-1}\right),
$$

If we now define:

$$
L=\operatorname{diag}\left(\sqrt{s_{1}}, \ldots, \sqrt{s_{2 L-1}}\right),,
$$

the total fluence is simply written as:

$$
F_{0}=\sum_{j=}^{2 L-1} \beta_{j}^{2},
$$

where:

$$
\beta=L U^{T} \alpha .
$$

Equation (34) tells us that the spaces of pulses with equal fluence are given by spheres in $\beta$-space. Therefore, we may finally define the $u$ control parameters as the $2 L-2$ hyperspherical angles of the sphere whose radius is $\sqrt{F_{0}}$.

The peak intensity of the initial pulse determines the fluence for the search space. Note, however, that the peak intensity of the optimized pulse will in general not be the same. I show, in the following, three cases corresponding to initial peak intensities (a) $I=1.1210^{14} \mathrm{Wcm}^{-2}$; (b) $I=7.1510^{13} \mathrm{Wcm}^{-2}$; and (c) $I=4.0210^{13} \mathrm{Wcm}^{-2}$. The optimization algorithm essentially necessitates 
consecutive forward and backward propagations, that are performed by making use of the self-consistent exponential midpoint rule [34], with a time step of 0.76 as. The procedure needs an average of 20 conjugate-gradient iterations to achieve a good maximum, which requires around 100 propagations. The resulting optimal pulses are displayed in Fig. 1, in real time in the left panels, and in the frequency domain in the right panels. The oscillator strength of the molecule is also displayed in the right panels, in order to show the dipole allowed excitations.

In all cases, the effect of the optimization is creating a pulse with a combination of those frequencies, right below the ionization potential (also shown in the plots with a vertical line). This shows how the pulses populate a combination of excited states in addition to coupling the system to the ionization continuum. The fact that this combination results in a maximum proves that it is more effective producing a dissociative force that a single channel. This is to be expected, as obviously a linear combination of states contain more optimization freedom than a single one. Note that one advantage of this scheme is that the optimization can be performed without prior knowledge of the excitations of the system.

The effect of these optimized pulses on the system is represented in Fig. 2. The plots in this figure display the resulting Ehrenfest-TDDFT dynamics: the internuclear distances on the left side panels, and the relative forces on the right side ones. The two cases (a) and (b) result in photo-dissociation, whereas the case (c), performed at a lower fixed fluence, results in an ample oscillation. In fact, all runs performed at even lower fluences resulted in no dissociation, whereas all runs performed at higher fluences resulted in faster nuclear separations; this is the reason to show these three runs corresponding to this "transition" intensity regime.

Overlayed on these dissociation graphs on the left side of Fig. 2 are the bound-states electronic population, approximated as the electronic density integrated over the simulation volume. The system looses aproximately 0.7 electrons in case (a), 0.6 electrons in case (b), and 0.5 electrons in case (c). As noted before, the dissociation target does not penalize ionization of the system, and therefore the process is to be understood as a combination of pure dissociation and dissociative ionization. The relevant role played by ionization could already be expected when looking at the power spectra of the optimal pulses, very close to the ionization potential, as shown on the right panels of Fig. 1 (note, however, that in the presence of a strong field the ionization potential is Stark shifted).

On the right side panels of Fig. 2, one can see the relative forces driving the internuclear distances. It is clear how this relative force is positive on average during the action of the pulse (shadowed region), since this is the target of the optimization procedure. The force is created by the electronic excitation, partly to bound excited states, and partly to the continuum. This excitation is enough to photo-dissociate the system in the first two cases. After the pulse, the forces average to zero in both (a) and (b), correspoding to a constant dissociating velocity. In case (c), after the rebound of the internuclear distance, the force 


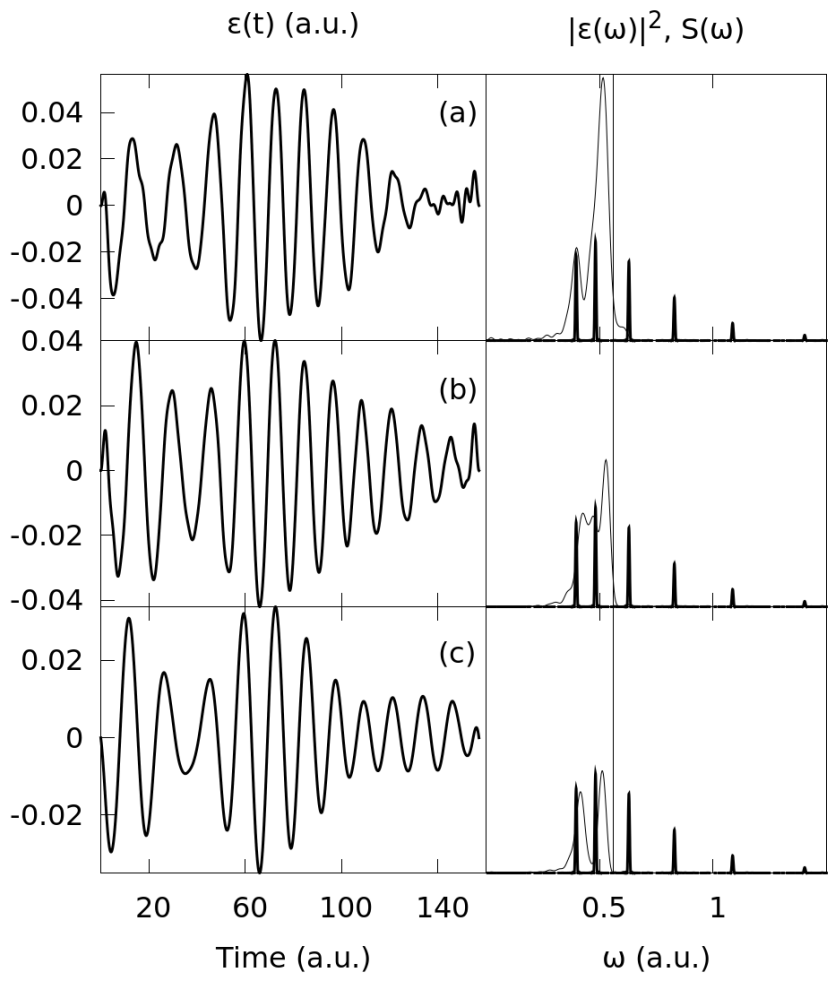

Figure 1: Optimized laser pulses in real time (left panels) and corresponding power spectra (right panels, thin curves), for three runs (a), (b) and (c) performed at different fixed fluences. The dipole strength function of the Hydrogen molecule is also displayed in thick lines on the right panels. The vertical line on the right panels shows the value of the ionization potential. 


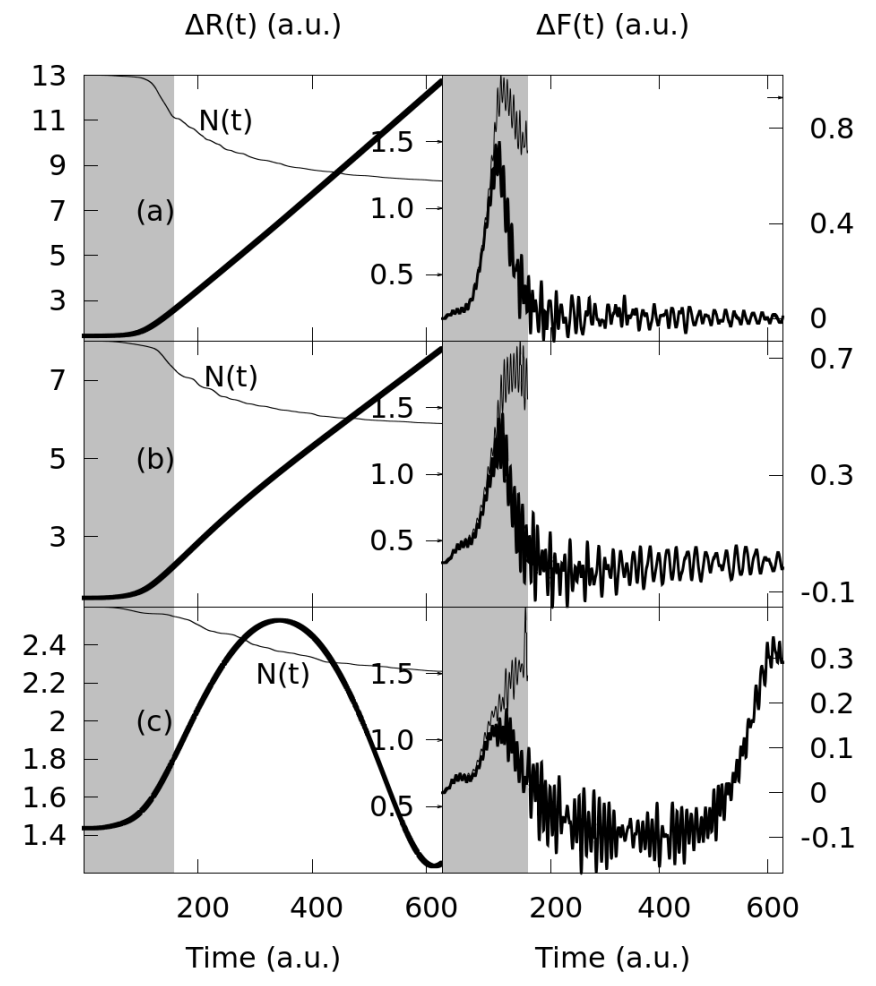

Figure 2: Internuclear distance (left panels) and relative forces (right panels) for the three runs (a), (b) and (c) performed at different fixed fluences. In the left panels, the total charge $\mathrm{N}(\mathrm{t})$ contained in the simulation region is also overlayed (thin lines, scale of the $y$-axis on the right side of each plot). In the right panels, the relative force computed with clamped nuclei is also displayed (lighter curves).

The shadowed region marks the time during which the pulse acts on the system. 


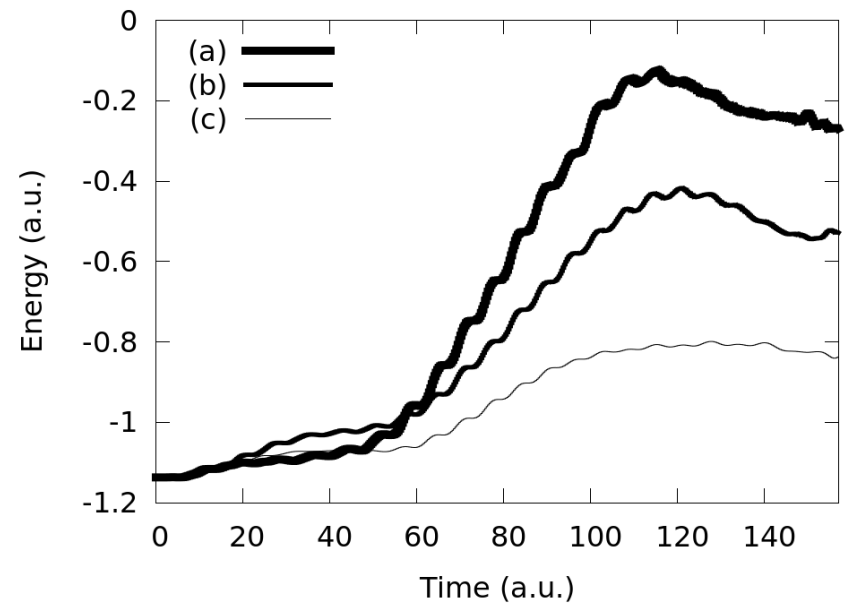

Figure 3: Time-dependent total energy of the molecule for the three pulses obtained with the the three optimization runs (a), (b) and (c) performed at different fixed fluences.

becomes positive again in order to drive the system towards the equilibrium position.

In Fig. 3, the total energies of the molecular system for the three pulses obtained with the the three optimization runs (a), (b) and (c) are displayed. The variation of the total electronic energy defined as the time derivative of the expectation value of the electronic Hamiltonian can be conveniently computed within TDDFT as:

$$
\frac{\mathrm{d}}{\mathrm{d} t} E(t)=\frac{\mathrm{d}}{\mathrm{d} t}\langle\Psi(t)|\hat{H}(t)| \Psi(t)\rangle=\frac{\mathrm{d} \varepsilon}{\mathrm{d} t}(t) \int \mathrm{d}^{3} r n(\vec{r}, t) \vec{r} \cdot \vec{p},
$$

i.e. the product of the time-derivative of the magnitude of the electric field, times the dipole moment of the system in the polarization direction of the field, $\vec{p}$. The total energy can be computed by integrating in time this term, and adding the nuclear classical kinetic and potential terms. Notice that the amount of energy deposited in the system is in all three cases is above the atomization energy of the system (around 0.13 a.u. in our model), and, as expected, increases with increasing fluence. In case (c), however, this does not suffice to produce dissociation, as part of the energy may be used to occupy excited bound states, or is taken away by the ionizing electrons.

Therefore, when sufficiently high fluences are allowed, the optimization scheme does its job and achieves the target. This happens despite the specially challenging choice of system and target: it becomes obvious from the plots that, even though the pulses have extremely short duration (approximately $3 \mathrm{fs}$ ), some ionic movement already occurs before the end of them. This fast reaction is due to both the light weight of the Hydrogen atoms, and the fact that the target 
is precisely chosen to enhance this movement. However, the present methodology only considers the electronic subsystem, and therefore the nuclear reaction necessarily implies a problem for the optimization: this is apparent in the right panels of Fig. 2, where both the relative atomic force computed with and without clamped nuclei are shown. This force, as computed during the optimization run, with clamped nuclei (displayed in lighter grey), is very strongly positive in the last femtosecond of the pulse duration, whereas the same force computed within the Ehrenfest-TDDFT simulation, is reduced in that time interval. The use of longer pulses, and the analysis and optimization of processes that involve nuclear movement, will require the generalization of this scheme to a theory that includes both electronic and nuclear degrees of freedom. This will be the subject of a forthcoming publication [35].

\section{Conclusions and Prospects}

The behavior of a many-electronic system in an external field may be optimized with respect to a predefined target by modeling it with TDDFT and employing the methodology of quantum optimal control in order to find the optimal field. The resulting equations are numerically tractable, as proved in this work in which the chosen system is a Hydrogen molecule and the target is defined in terms of the time-average of the forces acting on the nuclei. The computations were performed on modest, desktop type, computers, which allows to predict the applicability of these algorithms for larger systems, given the good scaling properties of TDDFT.

The theory presented in Ref. [4] and applied here addresses electronic-only systems. It is therefore directly applicable to explore the optimization possibilities in problems that do not involve significant nuclear movement - such as, for example, the problems already addressed for single or independent electrons: currents in quantum rings [19], manipulation of charge in quantum dots [20], and atomic of molecular ionization [21].

It may also be applied, as in this work, to trigger molecular re-arrangements through the use of ultrafast, few-femtosecond, pulses during which the ions do not have time for large movements - but whose action on the electronic system ultimately produces the final outcome. This sequential mechanism has been demonstrated for the photo-dissociation of the Hydrogen molecule by fewfemtosecond laser pulses. However, the very same examples described here have also been selected because they show the obvious caveat of the methodology: the nuclei start to move, and the resulting forces, computed during the molecular dynamics test runs, do not exactly correspond to the ones that were computed

during the optimization propagations. In order to correct for this problem, the solution is to design a control theory that dynamically addresses both electronic and nuclear degrees of freedom. Within the molecular dynamics paradigm, that treats the nuclear system classically, this control theory must tackle a hybrid quantum-classical system - for example the Ehrenfest-TDDFT model that was also used here. This is work in progress and will be presented elsewhere. 


\section{Acknowledgments}

Financial support provided by the European Commission within the FP7 CRONOS project (ID 280879), and by by the research project FIS2009-13364-C02-01 (MICINN, Spain).

\section{Keywords}

Time-dependent density-functional theory, optimal control theory, attosecond physics, photo-dissociation.

\section{References}

[1] E. Runge and E.K.U. Gross. Density-functional theory for time-dependent systems. Physical Review Letters, 52(12):997-1000, 1984.

[2] Miguel A. L. Marques, Neepa T. Maitra, Fernando M. S. Nogueira, Eberhard K. U. Gross, and Angel Rubio, editors. Fundamentals of TimeDependent Density Functional Theory, volume 837 of Lecture Notes in Physics. Springer, Berlin Heidelberg, 2012.

[3] Constantin Brif, Raj Chakrabarti, and Herschel Rabitz. Control of quantum phenomena: past, present and future. New Journal of Physics, 12:075008, 2010.

[4] A. Castro, J. Werschnik, and E. K. U. Gross. Controlling the dynamics of many-electron systems from first principles: A combination of optimal control and time-dependent density-functional theory. Physical Review Letters, 109:153603, 2012.

[5] Ferenc Krausz and Misha Ivanov. Attosecond physics. Rev. Mod. Phys., 81:163-234, Feb 2009.

[6] A Scrinzi, M Yu Ivanov, R Kienberger, and D M Villeneuve. Attosecond physics. Journal of Physics B: Atomic, Molecular and Optical Physics, 39(1):R1, 2006.

[7] M. Hentschel, R. Kienberger, Ch Spielmann, G. A. Reider, N. Milosevic, T. Brabec, P. Corkum, U. Heinzmann, M. Drescher, and F. Krausz. Attosecond metrology. Nature, 414(6863):509-513, Nov 2001.

[8] R. Kienberger, E. Goulielmakis, M. Uiberacker, A. Baltuska, V. Yakovlev, F. Bammer, A. Scrinzi, Th Westerwalbesloh, U. Kleineberg, U. Heinzmann, M. Drescher, and F. Krausz. Atomic transient recorder. Nature, 427(6977):817-821, Feb 2004. 
[9] Gustavo Bruneto, Umberto de Giovannini, Alberto Castro, Jessica Walkenhorst, and Angel Rubio. Pump-probe photo-electron and absorption spectroscopy in the attosecond time-scale with time-dependent densityfunctional theory. Submitted, 2013.

[10] A. M. Weiner. Femtosecond pulse shaping using spatial light modulators. Review of Scientific Instruments, 71(5):1929-1960, 2000.

[11] U. Saalmann and R. Schmidt. Non-adiabatic quantum molecular dynamics: basic formalism and case study. Zeitschrift für Physik, 38:153-163, 1996.

[12] F. Calvayrac, P.-G. Reinhard, and E. Suraud. Coulomb explosion of an na $_{12}$ cluster in a diabatic electronion dynamical picture. J. Phys. B: At. Mol. Opt. Phys., 31:5023-5030, 1996.

[13] Thomas Kunert and Rüdiger Schmidt. Excitation and fragmentation mechanisms in ion-fullerene collisions. Phys. Rev. Lett., 86:5258-5261, Jun 2001.

[14] A. Castro, M.A.L. Marques, J.A. Alonso, G.F. Bertsch, and A. Rubio. Excited states dynamics in time-dependent density functional theory. The European Physical Journal D - Atomic, Molecular, Optical and Plasma Physics, 28:211-218, 2004.

[15] Yoshiyuki Miyamoto and Hong Zhang. Electronic excitation in an $\mathrm{Ar}^{7+}$ ion traversing a graphene sheet: Molecular dynamics simulations. Phys. Rev. B, 77:161402, Apr 2008.

[16] A. Castro, M. Isla, Jos I. Martnez, and J.A. Alonso. Scattering of a proton with the li4 cluster: Non-adiabatic molecular dynamics description based on time-dependent density-functional theory. Chemical Physics, 399(0):130 $-134,2012$.

[17] Miguel A.L. Marques, Alberto Castro, George F. Bertsch, and Angel Rubio. octopus: a first-principles tool for excited electronion dynamics. Computer Physics Communications, 151(1):60 - 78, 2003.

[18] Alberto Castro, Heiko Appel, Micael Oliveira, Carlo A. Rozzi, Xavier Andrade, Florian Lorenzen, M. A. L. Marques, E. K. U. Gross, and Angel Rubio. octopus: a tool for the application of time-dependent density functional theory. physica status solidi (b), 243(11):2465-2488, 2006.

[19] E. Räsänen, A. Castro, J. Werschnik, A. Rubio, and E. K. U. Gross. Optimal control of quantum rings by terahertz laser pulses. Phys. Rev. Lett., 98:157404, Apr 2007.

[20] E. Räsänen, A. Castro, J. Werschnik, A. Rubio, and E. K. U. Gross. Optimal laser control of double quantum dots. Phys. Rev. B, 77:085324, Feb 2008. 
[21] A. Castro, E. Räsänen, A. Rubio, and E. K. U. Gross. Femtosecond laser pulse shaping for enhanced ionization. EPL (Europhysics Letters), 87(5):53001, 2009.

[22] Kevin Krieger, Alberto Castro, and E.K.U. Gross. Optimization schemes for selective molecular cleavage with tailored ultrashort laser pulses. Chemical Physics, 391(1):50 - 61, 2011. ¡ce:title ¿Open problems and new solutions in time dependent density functional theoryi/ce:title $\dot{i}$.

[23] Alberto Castro and Eberhard Gross. Quantum optimal control. In Miguel A.L. Marques, Neepa T. Maitra, Fernando M.S. Nogueira, E.K.U. Gross, and Angel Rubio, editors, Fundamentals of Time-Dependent Density Functional Theory, volume 837 of Lecture Notes in Physics, pages 265-276. Springer Berlin / Heidelberg, 2012. 10.1007/978-3-642-23518-4_13.

[24] W. Kohn. Nobel lecture: Electronic structure of matter ${ }^{-}$wave functions and density functionals. Rev. Mod. Phys., 71:1253-1266, Oct 1999.

[25] W. Kohn and L. J. Sham. Self-Consistent Equations Including Exchange and Correlation Effects. Physical Review, 140:1133-1138, November 1965.

[26] Miguel A.L. Marques, Micael J.T. Oliveira, and Tobias Burnus. Libxc: A library of exchange and correlation functionals for density functional theory. Computer Physics Communications, 183(10):2272 - 2281, 2012.

[27] J. P. Perdew and Alex Zunger. Self-interaction correction to densityfunctional approximations for many-electron systems. Phys. Rev. B, 23:5048-5079, May 1981.

[28] O. V. Gritsenko, S. J. A. van Gisbergen, A. Gorling, and E. J. Baerends. Excitation energies of dissociating $\mathrm{h}[\mathrm{sub} 2]$ : A problematic case for the adiabatic approximation of time-dependent density functional theory. The Journal of Chemical Physics, 113(19):8478-8489, 2000.

[29] Esa Räsänen and Lars Bojer Madsen. Strong-field-ionization suppression by light-field control. Phys. Rev. A, 86:033426, Sep 2012.

[30] R. Fletcher. Practical Methods of Optimization. Wiley, New York, 2000.

[31] M. Galassi, J. Davies, J. Theiler, B. Gough, G. Jungman, P. Alken, M. Booth, and F. Rossi. GNU Scientific Library. Network Theory Limited, 2009.

[32] Xavier Andrade, Joseba Alberdi-Rodriguez, David A Strubbe, Micael J T Oliveira, Fernando Nogueira, Alberto Castro, Javier Muguerza, Agustin Arruabarrena, Steven G Louie, Aln Aspuru-Guzik, Angel Rubio, and Miguel A L Marques. Time-dependent density-functional theory in massively parallel computer architectures: the octopus project. Journal of Physics: Condensed Matter, 24(23):233202, 2012. 
[33] N. Troullier and J. L. Martins. Phys. Rev. B, 43:1993, 1991.

[34] Alberto Castro, Miguel A. L. Marques, and Angel Rubio. Propagators for the time-dependent kohn-sham equations. The Journal of Chemical Physics, 121(8):3425-3433, 2004.

[35] A. Castro and E. K. U. Gross, in preparation. 